\title{
Highly-Sensitive Lock-in Thermography of Local Heat Sources Using 2-Dimensional Spatial Deconvolution
}

\author{
by O. Breitenstein, I. Konovalov, and M. Langenkamp
}

\author{
Max-Planck-Institut für Mikrostrukturphysik, Weinberg 2, D-06120 Halle, Germany, E-Mail: \\ breiten@mpi-halle.de \\ ABSTRACT:
}

A lock-in thermography system is introduced, which allows temperature modulations in the $10 \mu \mathrm{K}$ range to be detected. Under certain conditions (homogeneous infrared emissivity and thermal properties, good signal-to-noise ratio), the spatial resolution of lock-in thermographic investigations of local heat sources in electronic devices can be improved by spatial deconvolution. An iteratively working "vectorial" deconvolution procedure is presented, which takes into account the wave nature of the oscillating temperature field. Both heat sources lying at the surface and sources buried at a certain depth can be evaluated. The possibilities of this procedure are demonstrated by simulations and by the deconvolution of experimental lock-in thermograms.

\section{Introduction}

Lock-in thermography has become an established method for non-destructive device testing [1-3]. Recently, a novel lock-in thermography system has been developed, which is based on a $128 \times 128$ pixel mid-range (3-5 $\mu \mathrm{m}$ ) InSb focal plane array (FPA) infrared (IR) camera, running at a repetition rate of $217 \mathrm{~Hz}$ [4]. Performing the sin-/ cos-correlation on-line, this system shows a noise amplitude of about $10 \mu \mathrm{K}$ after a measure time of $1 / 2$ hour, thus guaranteeing a very good signal-to-noise ratio. As will be shown below, this is an important condition to successfully perform spatial deconvolution on lock-in thermograms.

The spatial resolution of the thermographic investigation of local heat sources is influenced by the lateral heat spreading in the sample, usually leading to a "halo" around local heat sources. For the lock-in thermography, the extension of this halo is governed by the frequency-dependent thermal diffusion length $\Lambda$, which in silicon is about $1 \mathrm{~mm}$ for $\mathrm{f}_{\text {lock-in }}=$ $30 \mathrm{~Hz}$ and decreases with $1 /{ } \mathrm{f}_{\text {lock-in. }}$. In order to minimize this thermal halo, a highest possible lock-in frequency should be applied. On the other hand, a high lock-in frequency leads to a reduced amplitude of the surface temperature modulation because of the heat capacity of the sample. Moreover, for practical reasons the maximum possible lock-in frequency is always limited. If a local heat source is at a certain depth below the surface of a solid, its lock-in thermogram generally appears blurred.

Since the temperature modulation field at the surface can always be interpreted as a convolution of a local lateral power distribution with a complex point spread function, it should be possible to reconstruct the lateral power distribution from measured thermograms numerically. This procedure is called deconvolution and has the potential to improve the spatial resolution of lock-in thermograms of local heat sources, if the thermal properties of the sample can be assumed to be homogeneous. Note that the locally varying phase of the thermal signal has to be considered here, because all sources are coherent and the thermal waves emerging from different local heat sources may interfere with each other. Moreover, it is well known that each spatial deconvolution procedure is strongly influenced by the noise and artifacts of the image. Therefore a highly sensitive lock-in thermography system is a necessary requirement for successfully applying spatial deconvolution. Thermographic artifacts, e.g. originating from an inhomogeneous IR emissivity, can be avoided by covering the sample with a black coating. 
The basic principles of our deconvolution software "VecDec" (Vectorial Deconvolution) had been introduced elsewhere [5] and will only briefly be reviewed here. Its first version allowed to evaluate only heat sources at the surface of the sample, and the thickness of the sample had to be below the thermal diffusion length $\Lambda$. Our latest version "VecDec 7" now also allows to evaluate thermally thick samples and heat sources lying at a certain depth below the surface. After reviewing the mathematical procedure of the iterative deconvolution procedure used here, a number of devonvolutions both for heat sources located at the surface and in a certain depth will be demonstrated. It will be shown how the algorithm reacts on certain parameter settings in order to find optimum deconvolution strategies for each application.

\section{The deconvolution procedure}

The most popular methods to perform 2-dimensional deconvolutions are the fourier transform method, working in fourier space, and iterative methods working in the real space [6]. Here we have chosen an iterative method because it provides more degrees of freedom (e.g. deconvolution only up to a certain degree or application of a positivity constraint condition, see below). Moreover, only this concept allows to work with phase-independent amplitude images, which are a non-linear combination of the two phase components of the temperature oscillation field. The procedure is based on a finite element approach with the network given by the pixel positions of the image. Hence, it is assumed that the measured oscillating temperature field at the surface of the sample is the sum of the influence of oscillating point heat sources in all pixel positions, yielding the power distribution $\mathrm{P}(\mathbf{r})$. Let the complex temperature modulation field in the position $\mathbf{r}=(\mathrm{x}, \mathrm{y})$ around one single unit heat point source located at $(0,0)$ be $\delta T(r)=\delta T^{\mathrm{re}}(\mathbf{r})+i \delta T^{\mathrm{im}}(\mathbf{r})$. The real part is the in-phase component and the imaginary part is the $90^{\circ}$ phase shifted component of the temperature oscillation. $\delta T(\mathbf{r})$ is called the complex point spread function (PSF). If there is a lateral distribution of heat point sources of the power $P(\mathbf{r})$, both the real and the imaginary temperature fields of all point sources superimpose linearly. Then the temperature field can be described as a numerical convolution of the point spread function $\delta \mathrm{T}(\mathbf{r})$ with the power distribution $\mathrm{P}(\mathbf{r})$ :

$$
\delta \mathrm{T}(\mathbf{r})=\sum_{\mathrm{r}^{\prime}} \mathrm{P}\left(\mathbf{r}^{\prime}\right) \delta \mathrm{T}\left(\mathbf{r}-\mathbf{r}^{\prime}\right)
$$

All summands in (1) with $\mathbf{r} \neq \mathbf{r}^{\prime}$ can be easily calculated, but for $\mathbf{r}=\mathbf{r}^{\prime}$ the summand would diverge if $\delta T(0)$ diverges, as it may do for the real part of the PSF. To avoid this divergence, $\delta \mathrm{T}(0)$ is calculated using the integral of the PSF across a circular region, which has the same area as one pixel with a pixel distance $\Delta$ has:

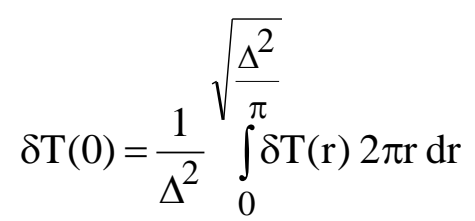

Physically (2) corresponds to the replacement of the point source by a homogeneous source within this pixel. If amplitude images have to be evaluated, the sum (1) has to be calculated separately for the real and the imaginary part of $\delta T(r)$ to obtain the in-phase $\left(0^{\circ}\right)$ and the quadrature $\left(90^{\circ}\right)$ image of the T-oscillation field. Then, in each position the phaseindependent amplitude image $A(r)$ and the phase image $\Phi(\mathbf{r})$ can be obtained using the wellknown relations:

$$
\mathrm{A}(\mathbf{r})=\sqrt{\left(\delta \mathrm{T}^{\mathrm{re}}(\mathbf{r})\right)^{2}+\left(\delta \mathrm{T}^{\mathrm{im}}(\mathbf{r})\right)^{2}} \quad \Phi(\mathbf{r})=\arctan \left(\frac{\delta \mathrm{T}^{\mathrm{im}}(\mathbf{r})}{\delta \mathrm{T}^{\mathrm{re}}(\mathbf{r})}\right)
$$


In principle, the deconvolution could be performed using either the $0^{\circ}$-image, the $90^{\circ}$ image, or the amplitude image. However, for a homogeneously heated thin sample area the temperature modulation is always $-90^{\circ}$ phase shifted to the power, hence the $0^{\circ}$ image contains no information in this case. Therefore our procedure allows to deconvolute only amplitude- and $-90^{\circ}$ images. The $-90^{\circ}$ image is preferred to the $+90^{\circ}$ one because only the first one is essentially positive in sign.

The actual deconvolution procedure for obtaining the power distribution $P(\mathbf{r})$ from one image $T(\mathbf{r})$ consists in performing the following steps:

1. As the first approximation for the power distribution $P_{1}(r)$ a distribution proportional to the measured image is taken.

$$
\mathrm{P}_{1}(\mathbf{r}) \propto \delta \mathrm{T}(\mathbf{r})
$$

2. From this power distribution the (first approximation) temperature field $\delta T_{1}(\mathbf{r})$ is calculated using eqs. (1)-(3), which usually differs from the measured one $\delta T(\mathbf{r})$. If instead of a $-90^{\circ}$ image an amplitude image is used, both the $0^{\circ}$ and the $90^{\circ}$ temperature field have to be calculated to get the amplitude image according to eq. (3).

3. For each pixel a power correction being proportional to the difference between the measured and the calculated T-distribution is calculated and subtracted from $\mathrm{P}_{1}(\mathbf{r})$, leading to the second approximation $\mathrm{P}_{2}(\mathbf{r})$

$$
\mathrm{P}_{2}(\mathbf{r})=\mathrm{P}_{1}(\mathbf{r})-\mathrm{m}\left(\delta \mathrm{T}_{1}(\mathbf{r})-\delta \mathrm{T}(\mathbf{r})\right)
$$

Here $\mathrm{m}$ is a scaling factor ("loop gain"), which can be used to optimize the convergency of the procedure.

4. On demand all appearing negative values of $P_{n}(r)$ can be set to zero. This so-called "positivity constraint" condition may be useful to suppress spatial oscillations in the result.

5. Steps 2-4 are repeated until a satisfactory convergence of the procedure is reached.

The convergence of this procedure relies the fact that, though (5) makes also corrections at the wrong positions, in the source positions these corrections will be the largest, since the PSF always has its maximum at $(0,0)$. It can be hoped that after a sufficient number of iterations these "favourable" corrections will dominate the not favorable ones. If the original power distribution is correctly reconstructed by the process, $\delta \mathrm{T}_{\mathrm{n}}=\delta \mathrm{T}$ will hold, all corrections in (5) will become zero, and the process will reach its stable convergence. This procedure is implemented in the "VecDec" deconvolution software, which is available from the authors. One of the goals of this development was to treat both spatially extended and local heat sources. Extended sources, however, cause problems if they cross the edge region of the image, since then also the region outside of the image contributes to the $T$-modulation within the image. We have solved this problem by "mirroring" the heat sources from inside the image to outside of it. This procedure is also advantageous if the image just fills the sample area, since also here the sample edges prevent lateral outdiffusion of heat, thus acting as a "heat mirror". If, however, there are real local heat sources besides the image region, this may lead to artifacts in the result.

The degrees of freedom in using the VecDec program are the following:

1. Either the amplitude- or the $-90^{\circ}$-image can be used for deconvolution.

2. The loop gain $m$ can be selected. The larger $m$ is, the faster is the convergence of the procedure. However, if $\mathrm{m}$ is too large, the solution oscillates from iteration to iteration.

3. The thermal diffusion length can be selected or calculated from the material parameters and from the lock-in frequency chosen within the program.

4. It can be selected whether the thermal waves spread only laterally in 2 dimensions (cylinder symmetry, corresponding to a thin sample relative to the thermal diffusion length $\Lambda$ ), or whether they spread 3-dimensionally (spherical symmetry, corresponding to a thick sample). In the latter case the depth of the heat sources can be selected.

5 . It can be chosen whether the positivity constraint condition will be applied or not. 
6. The size of the PSF matrix can be selected. The larger this size is, the higher gets the calculation time, but the more accurate is the calculation. The program suggests an optimum size.

7. The number of iterations can be selected. This selection allows to choose a compromise between the degree of deconvolution and the noise enhancement, see example below.

\section{Results}

\subsection{Simulations}

In order to test the general behaviour of the procedure, simulated thermograms are deconvoluted first. Hence, using (1) - (3) from a given power distribution the temperature modulation fields (amplitude or $-90^{\circ}$ ) are calculated, 1\% of statistical noise (referred to the maximum signal) was added, and these thermograms have been deconvoluted. For the first simulation we have assumed a thermally thin sample (thickness « $\Lambda$ ) where any depth gradient of $\mathrm{T}$ can be neglected, hence 2-dimensional heat spreading has been assumed. The thermal diffusion length $\Lambda$ has been chosen to be 4 pixel distances, and the size of the PSF matrix is 20 pixels. The model sample contains one extended area of homogeneous heating, one single pixel heat source, and a one pixel wide line source. All power densities are equal. Fig. 1 shows the original heat source, the simulated $-90^{\circ}$ image, and deconvoluted power distributions after 10, 30, 100 and 300 iteration cycles. The positivity constraint condition was applied in all cases. We see that the noise increases as the degree of deconvolution increases, as expected. However, even the tiny point source, which was practically invisible in the thermogram (b), clearly appears after deconvolution. The deconvolution restores the spatial resolution down to the pixel size. This demonstrates the gain in information that may be obtained using spatial deconvolution even on noisy lock-in thermography images.

Fig. 2 summarizes the results of the deconvolution of simulated images for the same power distribution in the case of an amplitude image in a thin sample and for the case of a thick sample with the heat sources located 4 pixel distances $(=\Lambda)$ deep below the surface. Simulations for a thick sample with heat sources at the surface have also been performed. They look similar to that of a thin sample, Fig. 3 shows one practical example of this type. For the examples in Fig. 2 the thermal diffusion length $\Lambda$ was chosen again to be 4 pixels, $1 \%$ of statistical noise was added to all thermograms, and 100 iterations have always been used. We see that for the amplitude images the deconvolution is less influenced by the noise than for the $-90^{\circ}$ images. The local heat sources are better resolved in Fig. 2 (b) than in Fig. 1 (e). This is because the PSF of the amplitude has a sharper peak at $(0,0)$, since it contains the $0^{\circ}$ signal, which actually diverges at $(0,0)$. On the other hand, the PSF of the buried heat sources is blurred most strongly, leading to the least efficient deconvolution in these cases. Interestingly, the deconvolution of amplitude images leads in both cases to some spurious heat sources in the region between the real heat sources. This seems to be an artifact of the procedure itself, stemming from the fact that for the calculation of the amplitude the phase information is lost. However, these artifacts appear only at the edge of a sharply defined region of homogeneous heat generation. In most practical cases they do not appear, as the practical examples in the following chapter will show.

\subsection{Deconvolution of experimental images}

A lock-in thermogram (amplitude image) of a $1.7 \times 1.7 \mathrm{~mm}^{2}$ sized region of a silicon solar cell under $0.5 \mathrm{~V}$ pulsed forward bias is shown in Fig. 4 (a). Here the lock-in frequency was 54 $\mathrm{Hz}$ and the pixel distance is $13 \mu \mathrm{m}$. Hence, 3-dimensional heat spreading with the heat source located at the surface has to be considered here. After 30 iterations (b) the halos are effectively removed. The resulting diameter of the heat sources is mainly governed by a 20 $\mu \mathrm{m}$ thick black plastic film, which had covered the surface of this cell. In the final example (Fig. 4) the amplitude image and the deconvoluted lateral power distribution of a special 
ultrasonic (US) test device is shown. In this device a horizontal $2 \mathrm{~mm}$ diameter hole, located $2 \mathrm{~mm}$ below the surface of an Al block, was filled with metal particles and exposed to US of $20 \mathrm{kHz}$, which was square-wave amplitude modulated at $0.85 \mathrm{~Hz}$. Some part of the US energy is converted into heat by the metal particles. The diameter of the buried heat source is correctly reconstructed by the deconvolution procedure. Here, the known average depth of the source was used for the calculation. The region without any noise beside the heat source is probably due to the fact that the depth position of the heat source is not constant here.

\section{Conclusion}

An iterative procedure for improving the spatial resolution of lock-in thermograms by 2dimensional spatial deconvolution has been introduced. Requirement for its application is that the IR emissivity is constant across the investigated region, so that the lock-in thermogram really reflects the distribution of the local surface T-modulation. The corresponding software "VecDec" running under Windows 95 / 98 / NT is available from the authors. Our simulations have shown that for amplitude images the statistical noise is less disturbing than for $-90^{\circ}$ images. While for heat sources located at the surface the attainable spatial resolution may come into the order of one pixel, the reconstruction of heat sources below the surface is less accurate. Under special conditions the deconvolution of amplitude images may lead to characteristic artifacts, which, however, do not play a role in practical applications.

\section{References}

[1] KUO, P. K., AHMED, T., HUIJIA, JIN, and DETROIT, M. I., "Phase-locked image acquisition in thermography", Proc. Automated Inspection and High Speed Vision Architectures II, Cambridge MA (1988), Proc. SPIE 1004 (1989), pp. 41-45

[2] BUSSE, G., WU, D., and KARPEN, W., "Thermal wave imaging with phase sensitive modulated thermography", J. Appl. Phys. 71, 3962 (1992)

[3] BALAGEAS, D. L., LEVESQUE, P., and DÉOM, A., "Characterization of electromagnetic fields using a lock-in infrared thermographic system", Proc. Thermosense II, Orlando 1993, Proc. SPIE 1933 (1993), pp. 274-285

[4] BREITENSTEIN, O., LANGENKAMP, M., ALTMANN, F., KATZER, D., LINDNER, A., and EGGERS, H., "Microscopic lock-in thermography investigation of leakage sites in integrated circuits", Rev. Sci. Instr., in print

[5] KONOVALOV, I. and BREITENSTEIN, O., "Evaluation of thermographic investigations of solar cells by spatial deconvolution", 2nd World Conf. on Photovolt. Energy Conversion, Vienna 1998, Proceedings pp. 148-151. See also: KONOVALOV, I.," Zusammenhang zwischen elektrischen Verlustmechanismen und der Struktur von Solarzellen", Dissertation Halle 2000, Der Andere Verlag, Osnabrück (2000)

[6] DEMOMENT, G., "Image reconstruction and restoration: Overview of common estimation structures and problems", IEEE Trans. on Acoust., Speech, and Sign. Proc., 37, 2024 (1989)

\section{Figures:}

Fig. 1: Original assumed power distribution (a), simulated lock-in thermogram $\left(90^{\circ}\right.$ image with $1 \%$ noise added, b), and deconvoluted power distributions after 10 (c), 30 (d), 100 (e) and 300 iterations (f) 

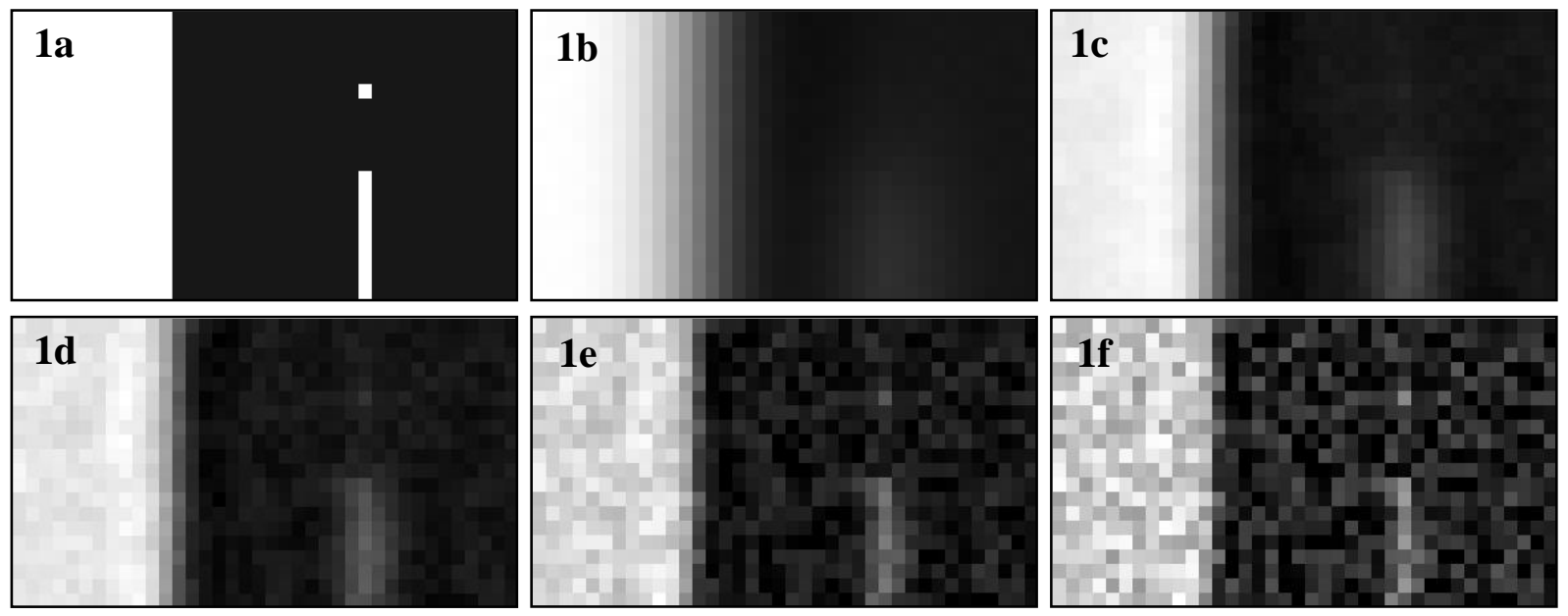

Fig. 2: Simulated lock-in thermograms $(\mathrm{a}, \mathrm{c}, \mathrm{e})$ and power distributions deconvoluted after 100 iterations $(b, d, f)$. The assumed power distribution is that of Fig. 1 (a). In all cases $1 \%$ statistical noise (referred to the maximum signal) was added to the thermograms. $(a, b)$ : amplitude image, 2dimensional heat spreading; (c,d): $-90^{\circ}$ image, 3-dimensional heat spreading, source depth: 4 pixels; $(\mathrm{e}, \mathrm{f})$ : amplitude image, 3dimensional heat spreading, source depth: 4 pixels.

Fig. 3: Lock-in thermogram (amplitude image, a) and deconvoluted power distribution (30 iterations, b) of a group of shunts in a Si solar cell. Size of the imaged region: $1.7 \times 1.7 \mathrm{~mm}^{2}$.

Fig. 4: Lock-in thermogram $2 \mathbf{a}$
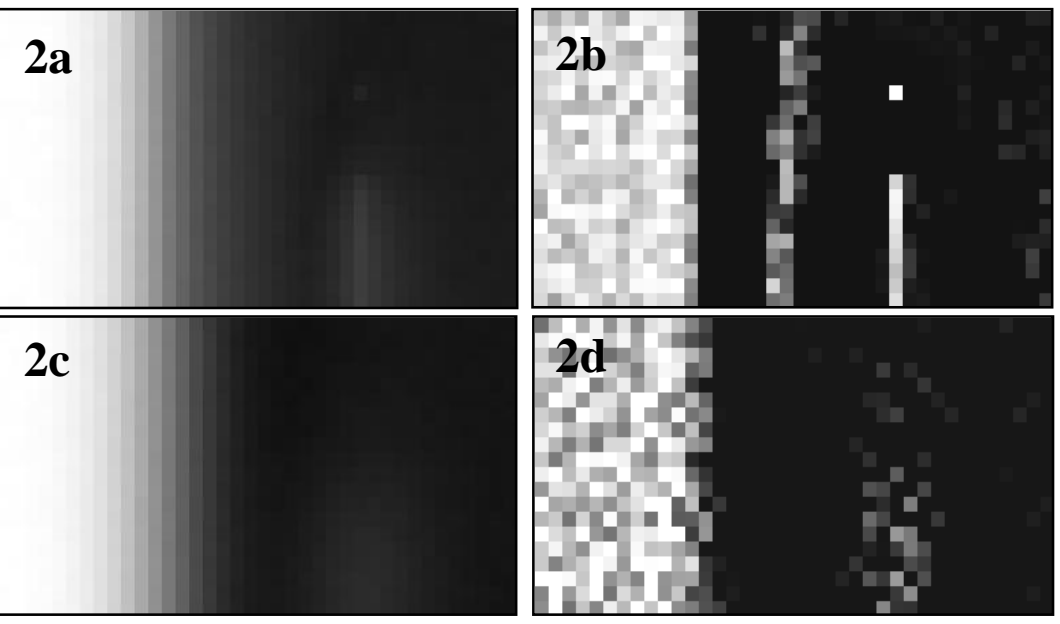

$2 e$
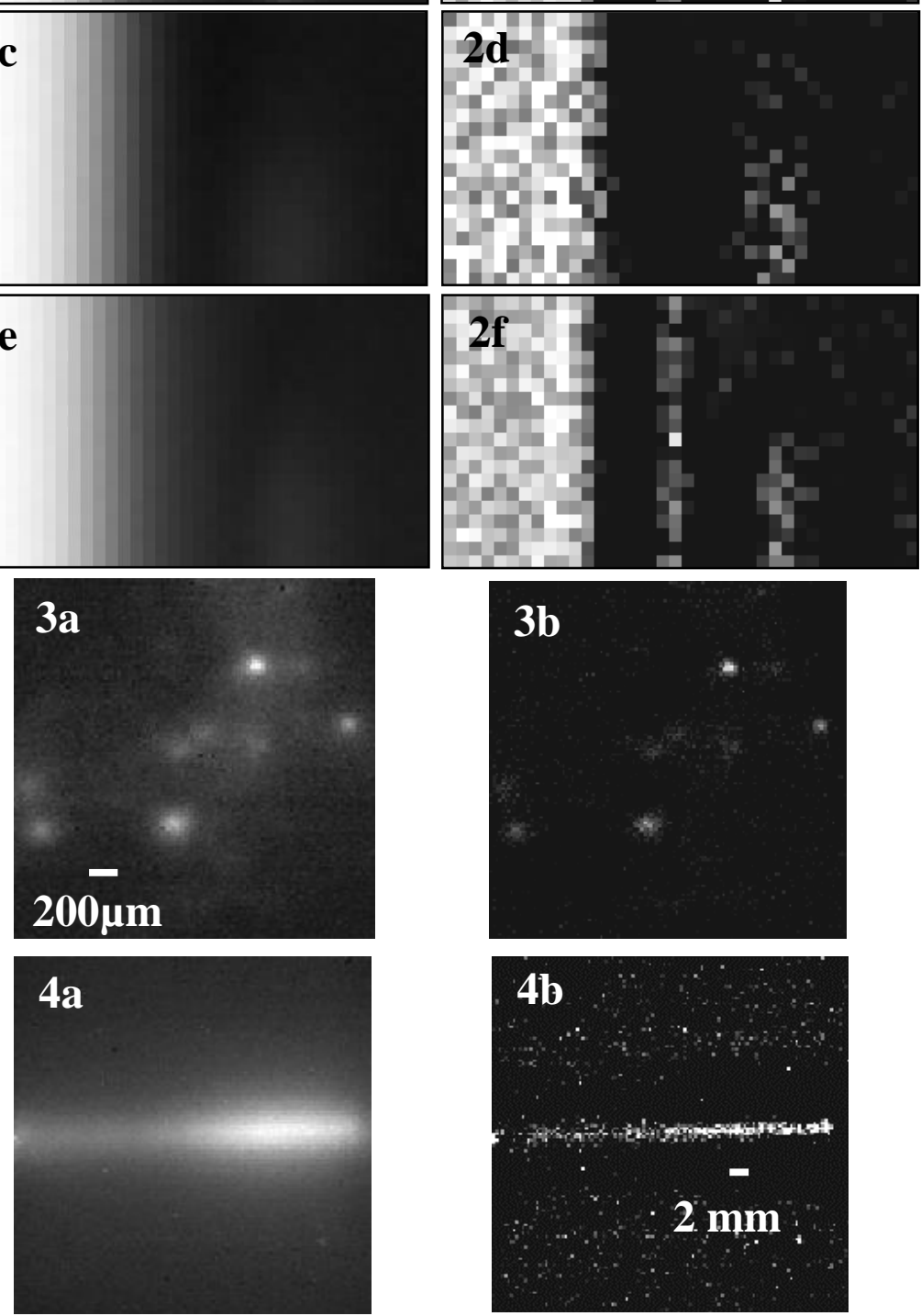

(amplitude image, a) and deconvoluted power distribution (30 Iterations, b) of an US test device containing a heat source buried at a depth of 2 $\mathrm{mm}$ below the surface 